\title{
The Issue of Subclinical Leaflet Thrombosis After Transcatheter Aortic Valve Implantation
}

\author{
Dinaldo C. Oliveira ${ }^{\mathrm{a}, \mathrm{e}}$, Sercan Okutucu ${ }^{\mathrm{b}}$, Giulio Russo ${ }^{\mathrm{c}}$, \\ Estevao C. Campos Martins ${ }^{\mathrm{d}}$
}

\begin{abstract}
Transcatheter aortic valve implantation (TAVI) has been considered an important therapy for the treatment of symptomatic severe aortic stenosis. Although the devices and the techniques have been improved some complications may occur and several issues still need to be addressed. The issue of subclinical leaflet thrombosis (SLT) has been recognized as a complication after TAVI, and its incidence ranges from $0 \%$ to $40 \%$. Nowadays, computed tomography is considered as the standard method for diagnosis of SLT. The concept of hypoattenuated leaflet thickening (HALT), reduced leaflet motion (RELM), and hypoattenuation affecting motion (HAM) have been used in this topic. Most patients who had SLT were taking single or dual antiplatelet therapy. In addition, these medications were not effective in resolving this complication after TAVI. However, there is a suggestion that oral anticoagulants have a protective and therapeutic effect. With the increasing use of TAVI, it is necessary to have better knowledge about several aspects of this complication, because it may have impact on prognosis. Therefore, some aspects of SLT diagnosis, management, and prognosis are not yet fully understood.
\end{abstract}

Keywords: Aortic stenosis; Subclinical leaflet thrombosis; Oral anticoagulants; Dual antiplatelet therapy

\section{Introduction}

Transcatheter aortic valve implantation (TAVI) has been considered an important therapy for the treatment of symptomatic severe aortic stenosis. Initially used for patients considered inoperable or at high risk, this therapy subsequently demonstrated its usefulness in patients at intermediate and low risk

Manuscript submitted May 20, 2020, accepted June 5, 2020

Published online August 1, 2020

${ }^{a}$ Federal University of Pernambuco, Recife, PE, Brazil

bMemorial Hospital Ankara, Ankara, Turkey

${ }^{c}$ Universita Cattolica del Sacro Cuore, Roma, Italy

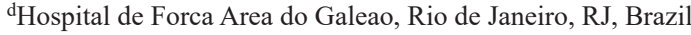

${ }^{e}$ Corresponding Author: Dinaldo C. Oliveira, Federal University of Pernam-

buco, Recife, PE, Brazil. Email: dinaldo@cardiol.br

doi: https://doi.org/10.14740/cr1108 for aortic valve replacement surgery $[1,2]$.

With the evolution of devices and new generation prostheses, the major complications of TAVI have been reduced. Although the long-term durability of these bioprostheses is still not known, mid-term data reveal a low rate of structural degeneration of bioprosthesis [3]. However, the issue of subclinical leaflet thrombosis (SLT) has been associated with some concerns. There is a necessity of better understanding this entity and new data are waited.

\section{Frequency of SLT}

Several technical and clinical aspects of TAVI have been better understood in recent years. However, a study by Makkar et al [4] described that in a trial, 22 out of 55 patients $(40 \%)$ had SLT, and 17 out of 132 patients from registries (13\%) also had SLT. The scientific community has recognized this complication and started to carry out studies to better know and manage this event.

Leetmaa et al [5] evaluated 140 patients who underwent multidetector computed tomography (MDCT) 1 - 3 months after TAVI. The incidence of SLT was $4 \%$, and all patients showed normal echocardiograms. On the other hand, Pache et al [6] evaluated 156 patients who underwent TAVI and showed that the incidence of SLT was $10.3 \%$ (16 patients). Hansson et al [7] evaluated 405 patients who underwent MDCT (performed between 1 - 3 months) after TAVI and found that the incidence of SLT was 5.6\% (23 patients).

D'Ascenzo et al [8] performed a meta-analysis that selected 18 studies out of 291 that had identified thrombosis after TAVI. Seventeen studies were observational, and only one was randomized. Six studies enrolled 2,053 patients and showed an incidence of SLT as $1.36 \%$ per month and $16.32 \%$ per year.

Chakravarty et al [9] described a 13\% incidence of SLT in 752 patients who underwent TAVI and were enrolled in the RESOLVE and SAVORY registries. However, it should be noted that not all patients from these studies were evaluated, which means that 626 patients (71\%) from RESOLVE and $264(29 \%)$ from SAVORY were enrolled. In this study, the rates of non-ischemic stroke/transient ischemic attack (TIA) $(3 \%$ vs. $8 \%, \mathrm{P}=$ 0.004 (odds ratio (OR): 3.3 , confidence interval (CI): $1.45-7.5$ )) and post-computed tomography $(\mathrm{CT})$ stroke/TIA $(1 \%$ vs. $4 \%, \mathrm{P}=$ 0.04 (OR: 3.45, CI: 1.08 - 11.03)) were higher in those with SLT.

Makki et al [10] performed a meta-analysis of six studies that enrolled 1,560 patients who underwent TAVI. The incidence of SLT was $12.6 \%$ (198 patients). At the end of the first 
Table 1. Frequencies of Subclinical Leaflet Thrombosis According to Different Studies

\begin{tabular}{lll}
\hline Authors & Patients $(\mathbf{N})$ & SLT (N) \\
\hline Pache et al, 2013 [12] & 1 patient (case report) & First case \\
Makkar et al, 2015 [4] & 55 patients & 22 patients $(40 \%)$ \\
Makkar et al, 2015 [4] & 132 patients & 17 patients $(13 \%)$ \\
Leetmaa et al, 2015 [5] & 140 patients & 6 patients $(4 \%)$ \\
Pache et al, 2016 [6] & 156 patients & 16 patients $(10 \%)$ \\
Hansson et al, 2016 [7] & 405 patients & 23 patients $(5.3 \%)$ \\
Chakravarty et al, 2019 [9] & 752 patients & 97 patients $(13 \%)$ \\
Rashid et al, 2018 [11] & 1,156 patients & 134 patients $(11 \%)$ \\
Makki et al, 2018 [10] & 1,560 patients & 198 patients $(12 \%)$ \\
D'Ascenzo et al, 2019 [8] & 2,053 patients & 334 patients $(16 \%)$ \\
\hline
\end{tabular}

SLT: subclinical leaflet thrombosis

year, patients who had SLT were more likely to have a stroke or TIA (OR: 2.45, CI: $1.4-2.99, \mathrm{P}=0.04$ ) and valve degeneration (OR: 1.9 , CI: $1.46-2.48, \mathrm{P}=0.004)$. However, the analysis of SVD was based only on five studies (93 patients with SLT).

Another meta-analysis carried out by Rashid et al [11] evaluated 1,156 patients and showed an incidence of SLT of $11.6 \%$. In these patients, there was a greater chance of cerebrovascular events (OR: 3.38, CI: $1.78-6.41, \mathrm{P}<0.001$ ). Table 1 [4-12] shows some studies which revealed rates of SLT.

The issue of SLT has been recognized as a complication after TAVI, and its incidence ranges from $0 \%$ to $40 \%$ [13]. This variability is partly justified by the lack of uniformity in the definition of SLT in the initial studies. However, other aspects, such as the timing of CT, and data from sub-studies in which CT was available, thus not reflecting all patients, may also have contributed to this variability. Besides, the majority of these studies were retrospective, single-center, or sub-studies, and they were performed to generate hypotheses.

Nowadays, CT is considered as the standard method for diagnosis of SLT. The concept of hypoattenuated leaflet thickening (HALT) (Fig. 1), reduced leaflet motion (RELM), and hypoattenuation affecting motion (HAM) have been used in this topic [14]. When the leaflets have normal mobility, no hypoattenuating material is observed, and there is adequate coaptation in diastole, which is difficult to observe on CT. However, they are easy to visualize when mobility of leaflets is reduced [15]. The hypoattenuating material in the leaflets on CT is called HALT, and this can lead to reduced mobility (RELM). HAM is defined by the presence of HALT and RELM simultaneously [15].

Two-dimensional (2D) tomography with multiplanar reconstruction is used to evaluate the leaflets, and when HALT is present, 4D volume-rendering CT for mobility assessment (evaluation of possible RELM) should be performed. RELM assessment is not necessary in the absence of HALT, as the possibility of SLT is not considered in that scenario. RELM has been classified as not significant (reduction $<50 \%$ mobility) and significant $(\geq 50 \%)$. This percentage was calculated by dividing the width of the leaflets (distance from the free margin to the tip in the maximum opening) by half the diameter of the frame (distance from the frame margin to the line in the center of it) [15].

The mobility of the leaflets is considered normal when there is no RELM, according to CT. In the presence of reduced mobility, this has been classified as mild $(<50 \%)$, moderate $(50-70 \%)$, severe $(>70 \%)$, and immobile (100\%) (Fig. 2) $[15,16]$.

The natural history of SLT is not well known. The timing of SLT after TAVI, resolution of thrombus without treatment, and short, medium, and long-term consequences of the SLT, as well as the best therapeutic strategies for prevention and treatment are some examples of topics that need to be better known.

The majority of the patients are asymptomatic, and the

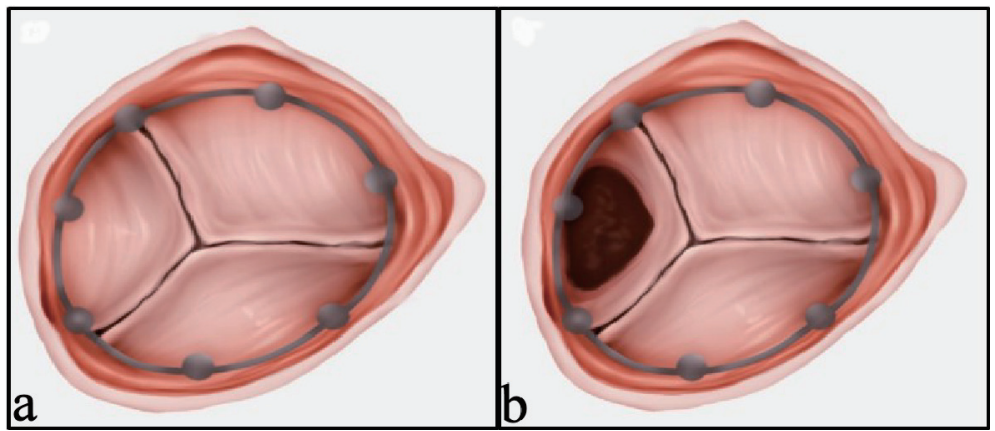

Figure 1. Hypoattenuated leaflet thickening (HALT). (a) HALT: negative. (b) HALT: positive. 


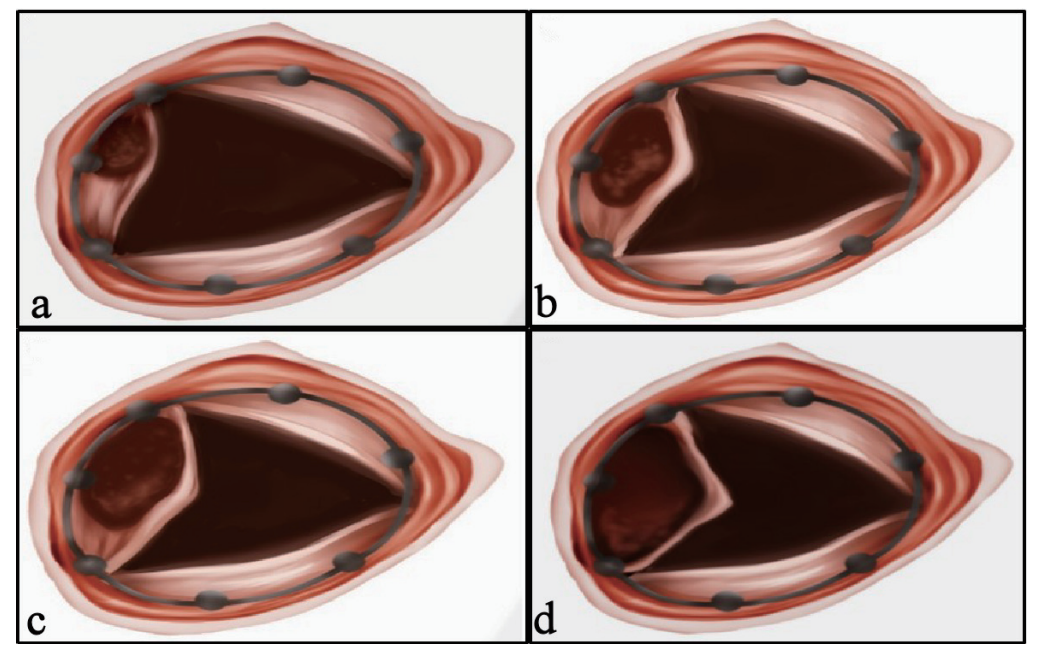

Figure 2. Reduced leaflet motion (RELM): classification. (a) Mild: < 50\%. (b) Moderate: $50-70 \%$. (c) Severe: $>70 \%$. (d) Immobile: $100 \%$.

gradient does not change, but D-dimer and N-terminal-pro-Btype natriuretic peptide (NT-proBNP) levels can be abnormal (increased) [16]. According to the most available data, SLT is an imaging finding [17].

Sub-studies of PARTNER 3 (transcatheter aortic valve replacement (TAVR) with balloon expandable valve in low-risk patients) and EVOLUT LOW RISK (TAVR with self expandable valve in low-risk patients) will clarify the relationship between HALT and neurological events [17].

The occurrence of HALT was detected at different time intervals after TAVI (mean: $159 \pm 177$ days, ranging from 21 to 596 days). In some cases, this finding remains stable; in others, it progresses to thrombosis, and in others, it regresses without specific treatment [17].

There have been hypotheses that SLT may increase the transvalvular gradient, reduce the durability of the prosthesis, and increase the chance of cerebrovascular events. However, these concerns are not based on data with strong scientific evidence $[16,17]$.

Therefore, SLT exists after TAVI; however, some aspects of its diagnosis, management, and prognosis are not yet fully understood. With the increasing use of TAVI, it is necessary to have better knowledge about several aspects of this complication.

\section{Pharmacology}

According to the guidelines, patients who undergo TAVI should be pre-treated with aspirin and clopidogrel, which should be maintained for up to 6 months. These initial recommendations were empirical; the knowledge of the impact of dual antiplatelet therapy (DAPT) on thrombotic events and the bleeding occurring in patients undergoing percutaneous coronary intervention are among the factors taken into consideration [18].

Some studies have questioned the need for DAPT as compared to single antiplatelet therapy (SAPT) since it was associated with a higher occurrence of bleeding without reducing ischemic events. However, most of these studies were observational, and therefore more robust evidence is needed [19-21].

SLT is a thrombotic phenomenon; therefore, the question arises whether DAPT or SAPT would be able to prevent this complication. In addition, the recognized efficiency of oral anticoagulants (OACs) in preventing and treating thrombosis of surgical valve prostheses has created a basis for the evaluation of such medications in this context $[22,23]$.

Pache et al [6] carried out a study (156 patients, SLT = $10 \%$ ) in which there was no difference in the rates of this complication between patients taking OACs, DAPT, or just one antiplatelet drug.

However, in the study by Makkar et al [4] (55 patients, $\mathrm{SLT}=40 \%$ ), it was found that in patients receiving vitamin $\mathrm{K}$ antagonist (VKA), the rate of this thrombotic event was zero, in contrast to those receiving DAPT $(51 \%, \mathrm{P}=0.001)$.

A study by Hansson et al [7] (405 patients) showed that OACs (vitamin K inhibitors) are associated with a lower SLT occurrence (1.8\% vs. $10.7 \%$, risk ratio (RR): 6.09 ; $95 \% \mathrm{CI}$ : $1.86-19.84)$.

Chakravarty et al [9] demonstrated that the use of OACs when compared to DAPT ( $4 \%$ vs. $15 \%, \mathrm{P}<0.001)$ and SAPT $(4 \%$ vs. $16 \%, \mathrm{P}<0.001)$ reduced the rates of SLT. In addition, there was no difference in the prevalence of this complication between double or single antiplatelet drugs (15\% vs. $15 \%, \mathrm{P}$ $=0.8$ ). Direct anticoagulants were as effective as vitamin $\mathrm{K}$ inhibitors (3\% (3 of 107) vs. 4\% (5 of 117), $\mathrm{P}=0.7$ ). SLT was resolved in $100 \%$ of patients receiving OAC (warfarin $67 \%$ and direct $\mathrm{OAC} 33 \%$ ), whereas it persisted in $91 \%$ of patients not receiving OAC $(\mathrm{P}<0.001)$.

Nuhrenberg et al [24] evaluated platelet reactivity in 200 patients receiving aspirin and clopidogrel who underwent CT 5 days after TAVI. This study revealed that DAPT did not modify the HALT findings, and platelet reactivity was not associated with this finding. On the other hand, OAC therapy reduced the occurrence of HALT.

Jimenez et al [25] published a study in which 85 patients underwent TAVI and CT, and there were 13 cases of SLT. Twelve 
of these patients received DAPT, and only one patient received OAC. This research also demonstrated that platelet reactivity had no impact on the occurrence of this complication.

Therefore, better data are an unmet need in this field. In this context, much better data are coming that will help us to understand the issue of SLT after TAVI.

Dangas et al [26] evaluated 1,664 patients without an established indication for OAC after TAVI, who received rivaroxaban (10 mg daily) or DAPT. After a median of 17 months, death or a first thromboembolic event had occurred in the rivaroxaban group (105 patients) and in the antiplatelet group (78 patients) (incidence rates: 9.8 and 7.2 per 100 personyears, respectively; hazard ratio with rivaroxaban: $1.35 ; 95 \%$ CI: $1.01-1.81 ; \mathrm{P}=0.04)$. Major, disabling, or life-threatening bleeding (intention-to-treat analysis) occurred in 46 and 31 patients, respectively, (4.3 and 2.8 per 100 person-years; hazard ratio: $1.50 ; 95 \% \mathrm{CI}: 0.95-2.37 ; \mathrm{P}=0.08)$. A total of 64 deaths occurred in the rivaroxaban group and 38 in the antiplatelet group (5.8 and 3.4 per 100 person-years, respectively; hazard ratio: $1.69 ; 95 \% \mathrm{CI}: 1.13$ - 2.53$)$.

Backer et al [27] evaluated a total of 231 patients. At least one prosthetic valve leaflet with grade 3 or higher motion reduction was found in two of 97 patients $(2.1 \%)$, who had scans that could be evaluated in the rivaroxaban group, compared with 11 of $101(10.9 \%)$ in the antiplatelet group (difference: $-8.8 \% ; 95 \% \mathrm{CI}:-16.5$ to $-1.9 ; \mathrm{P}=0.01)$. In a sub-study of a trial involving patients without an indication for long-term anticoagulation who had undergone successful TAVR, a rivaroxaban-based antithrombotic strategy was more effective than an antiplatelet-based strategy in preventing subclinical leaflet-motion abnormalities. However, in the main trial, the rivaroxaban-based strategy was associated with a higher risk of death or thromboembolic complications and a higher risk of bleeding than the antiplatelet-based strategy.

The AUREA [28] (DAPT versus oral anticoagulation for a short time to prevent cerebral embolism after TAVI) and AVATAR [29] (anticoagulation alone versus anticoagulation and aspirin following transcatheter aortic valve interventions) trials are also smaller-scale multicenter randomized studies comparing VKA with DAPT and aspirin plus VKA therapy for stroke and other complications.

Outcomes of AUREA trial has been presented in 2019. Initial results revealed that OACs following TAVR failed to lower the incidence of new subclinical cerebral lesions identified on imaging when compared to DAPT [30].

In summary, most patients who had SLT were taking DAPT or SAPT. In addition, these medications were not effective in resolving this complication after TAVI. However, there is a suggestion that OACs have a protective and therapeutic effect, although such evidence does not come from several randomized clinical studies. Several ongoing trials and studies will provide better data.

\section{Conclusions}

Occurrence of SLT is not a rare finding after TAVI, but the incidence varies according to intensity of screening. There is a debate if this finding has an impact on clinical events and on the durability of prostheses. DAPT or SAPT appears to be less effective for prevention and treatment of SLT than OACs. Ongoing clinical studies will contribute to the definition of which drug is the most effective for management of SLT.

\section{Acknowledgments}

The authors would like to thank Prof. Dr. Francesco Maisano, Prof. Dr. Maurizio Taramasso, Prof. Dr. Carlos Mestres, Assoc. Prof. Dr. Martin Andreas, other CAS-Aortic Valve Structural Interventions faculty and participants for enabling positive research and academic environment during writing process of this manuscript.

\section{Financial Disclosure}

None to declare.

\section{Conflict of Interest}

None to declare.

\section{Author Contributions}

All authors have collected data and written the article.

\section{Data Availability}

The authors declare that data supporting the findings of this study are available within the article.

\section{References}

1. Baumgartner H, Falk V, Bax JJ, De Bonis M, Hamm C, Holm PJ, Iung B, et al. 2017 ESC/EACTS Guidelines for the management of valvular heart disease. Eur Heart J. 2017;38(36):2739-2791.

2. Food and Drug Administration Expands TAVR indication to low risk patients. https:/www.acc.org/latest-incardiology/articles/2019/08/16/13/49/fda-expands-tavrindication-to-low-risk-patients.

3. Auffret V, Lefevre T, Van Belle E, Eltchaninoff H, Iung B, Koning R, Motreff P, et al. Temporal trends in transcatheter aortic valve replacement in France: FRANCE 2 to FRANCE TAVI. J Am Coll Cardiol. 2017;70(1):42-55.

4. Makkar RR, Fontana G, Jilaihawi H, Chakravarty T, Kofoed KF, De Backer O, Asch FM, et al. Possible subclinical leaflet thrombosis in bioprosthetic aortic valves. N Engl J Med. 2015;373(21):2015-2024.

5. Leetmaa T, Hansson NC, Leipsic J, Jensen K, Poulsen $\mathrm{SH}$, Andersen HR, Jensen JM, et al. Early aortic tran- 
scatheter heart valve thrombosis: diagnostic value of contrast-enhanced multidetector computed tomography. Circ Cardiovasc Interv. 2015;8(4).

6. Pache G, Schoechlin S, Blanke P, Dorfs S, Jander N, Arepalli CD, Gick M, et al. Early hypo-attenuated leaflet thickening in balloon-expandable transcatheter aortic heart valves. Eur Heart J. 2016;37(28):2263-2271.

7. Hansson NC, Grove EL, Andersen HR, Leipsic J, Mathiassen ON, Jensen JM, Jensen KT, et al. Transcatheter aortic valve thrombosis: incidence, predisposing factors, and clinical implications. J Am Coll Cardiol. 2016;68(19):20592069.

8. D'Ascenzo F, Salizzoni S, Saglietto A, Cortese M, Latib A, Franzone A, Barbanti M, et al. Incidence, predictors and cerebrovascular consequences of leaflet thrombosis after transcatheter aortic valve implantation: a systematic review and meta-analysis. Eur J Cardiothorac Surg. 2019;56(3):488-494.

9. Chakravarty T, Sondergaard L, Friedman J, De Backer O, Berman D, Kofoed KF, Jilaihawi H, et al. Subclinical leaflet thrombosis in surgical and transcatheter bioprosthetic aortic valves: an observational study. Lancet. 2017;389(10087):2383-2392.

10. Makki N, Shreenivas S, Kereiakes D, Lilly S. A metaanalysis of reduced leaflet motion for surgical and transcatheter aortic valves: Relationship to cerebrovascular events and valve degeneration. Cardiovasc Revasc Med. 2018;19(7 Pt B):868-873.

11. Rashid HN, Gooley RP, Nerlekar N, Ihdayhid AR, McCormick LM, Nasis A, Cameron JD, et al. Bioprosthetic aortic valve leaflet thrombosis detected by multidetector computed tomography is associated with adverse cerebrovascular events: a meta-analysis of observational studies. EuroIntervention. 2018;13(15):e1748-e1755.

12. Pache G, Blanke P, Zeh W, Jander N. Cusp thrombosis after transcatheter aortic valve replacement detected by computed tomography and echocardiography. Eur Heart J. 2013;34(46):3546.

13. Madukauwa-David ID, Sadri V, Midha PA, Babaliaros V, Aidun C, Yoganathan AP. An evaluation of the influence of coronary flow on transcatheter heart valve neo-sinus flow stasis. Ann Biomed Eng. 2020;48(1):169-180.

14. Sondergaard L, De Backer O, Kofoed KF, Jilaihawi H, Fuchs A, Chakravarty T, Kashif M, et al. Natural history of subclinical leaflet thrombosis affecting motion in bioprosthetic aortic valves. Eur Heart J. 2017;38(28):22012207.

15. Jilaihawi H, Asch FM, Manasse E, Ruiz CE, Jelnin V, Kashif M, Kawamori H, et al. Systematic CT methodology for the evaluation of subclinical leaflet thrombosis. JACC Cardiovasc Imaging. 2017;10(4):461-470.

16. Testa L, Latib A. Assessing the risk of leaflet motion abnormality following transcatheter aortic valve implantation. Interv Cardiol. 2018;13(1):37-39.

17. Mangieri A, Montalto C, Poletti E, Sticchi A, Crimi G, Giannini F, Latib A, et al. Thrombotic versus bleeding risk after transcatheter aortic valve replacement: JACC review topic of the week. J Am Coll Cardiol. 2019;74(16):2088-2101.

18. Rosseel L, De Backer O, Sondergaard L. Clinical valve thrombosis and subclinical leaflet thrombosis following transcatheter aortic valve replacement: is there a need for a patient-tailored antithrombotic therapy? Front Cardiovasc Med. 2019;6:44.

19. Nijenhuis VJ, Brouwer J, Sondergaard L, Collet JP, Grove EL, Ten Berg JM. Antithrombotic therapy in patients undergoing transcatheter aortic valve implantation. Heart. 2019;105(10):742-748.

20. Rodes-Cabau J, Masson JB, Welsh RC, Garcia Del Blanco B, Pelletier M, Webb JG, Al-Qoofi F, et al. Aspirin versus aspirin plus clopidogrel as antithrombotic treatment following transcatheter aortic valve replacement with a balloon-expandable valve: the ARTE (Aspirin Versus Aspirin + Clopidogrel Following Transcatheter Aortic Valve Implantation) randomized clinical trial. JACC Cardiovasc Interv. 2017;10(13):1357-1365.

21. Hassell ME, Hildick-Smith D, Durand E, Kikkert WJ, Wiegerinck EM, Stabile E, Ussia GP, et al. Antiplatelet therapy following transcatheter aortic valve implantation. Heart. 2015;101(14):1118-1125.

22. Nakatani S. Subclinical leaflet thrombosis after transcatheter aortic valve implantation. Heart. 2017;103(24):19421946.

23. Ruile P, Jander N, Blanke P, Schoechlin S, Reinohl J, Gick M, Rothe J, et al. Course of early subclinical leaflet thrombosis after transcatheter aortic valve implantation with or without oral anticoagulation. Clin Res Cardiol. 2017;106(2):85-95.

24. Nuhrenberg TG, Hromek J, Kille A, Hochholzer W, Hein M, Trenk D, Neumann FJ, et al. Impact of on-clopidogrel platelet reactivity on incidence of hypoattenuated leaflet thickening after transcatheter aortic valve replacement. JACC Cardiovasc Interv. 2019;12(1):12-18.

25. Jimenez C, Ohana M, Marchandot B, Kibler M, Carmona A, Peillex M, Heger J, et al. Impact of antithrombotic regimen and platelet inhibition extent on leaflet thrombosis detected by cardiac MDCT after transcatheter aortic valve replacement. J Clin Med. 2019;8:506-523.

26. Dangas GD, Tijssen JGP, Wohrle J, Sondergaard L, Gilard M, Mollmann H, Makkar RR, et al. A controlled trial of rivaroxaban after transcatheter aortic-valve replacement. N Engl J Med. 2020;382(2):120-129.

27. De Backer O, Dangas GD, Jilaihawi H, Leipsic JA, Terkelsen CJ, Makkar R, Kini AS, et al. Reduced leaflet motion after transcatheter aortic-valve replacement. N Engl J Med. 2020;382(2):130-139.

28. ClinicalTrials.gov. Dual antiplatelet therapy versus oral anticoagulation for a short time to prevent cerebral embolism after TAVI (AUREA). Available online: https:// clinicaltrials.gov/ct2/show/NCT01642134.

29. ClinicalTrials.gov. Anticoagulation alone versus anticoagulation and aspirin following transcatheter aortic valve interventions (1:1) (AVATAR). Available online: https:// clinicaltrials.gov/ct2/show/NCT02735902.

30. Diaz VAJ, in behalf of the AUREA investigators. Shortcourse dual antiplatelet therapy versus oral anticoagulation to prevent cerebral embolism after transcatheter aortic valve replacement. Presented at: TCT 2019. September 28, 2019. San Francisco, CA. 